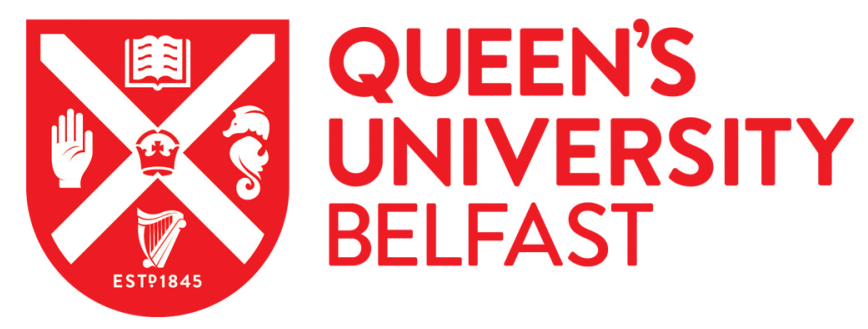

\title{
Intratumoral sterol-27-hydroxylase (CYP27A1) expression in relation to cholesterol synthesis and vitamin D signaling and its association with lethal prostate cancer
}

Khan, N. A., Stopsack, K., Allott, E. H., Gerke, T., Giovannucci, E. L., Mucci, L. A., \& Kantoff, P. W. (2019). Intratumoral sterol-27-hydroxylase (CYP27A1) expression in relation to cholesterol synthesis and vitamin D signaling and its association with lethal prostate cancer. Cancer Epidemiology Biomarkers \& Prevention, 28(6), 1052-1058. https://doi.org/10.1158/1055-9965.EPI-18-1083

Published in:

Cancer Epidemiology Biomarkers \& Prevention

Document Version:

Peer reviewed version

Queen's University Belfast - Research Portal:

Link to publication record in Queen's University Belfast Research Portal

Publisher rights

Copyright 2019 AACS. This work is made available online in accordance with the publisher's policies. Please refer to any applicable terms of use of the publisher.

\section{General rights}

Copyright for the publications made accessible via the Queen's University Belfast Research Portal is retained by the author(s) and / or other copyright owners and it is a condition of accessing these publications that users recognise and abide by the legal requirements associated with these rights.

Take down policy

The Research Portal is Queen's institutional repository that provides access to Queen's research output. Every effort has been made to ensure that content in the Research Portal does not infringe any person's rights, or applicable UK laws. If you discover content in the

Research Portal that you believe breaches copyright or violates any law, please contact openaccess@qub.ac.uk. 


\section{Intratumoral sterol-27-hydroxylase (CYP27A1) expression in relation to cholesterol synthesis and vitamin $\mathrm{D}$ signaling and its association with lethal prostate cancer}

Nabeela A. Khan, ${ }^{1 *}$ Konrad H. Stopsack, ${ }^{1,2 *}$ Emma H. Allott, ${ }^{2,3}$ Travis A. Gerke, ${ }^{2,4}$

Edward L. Giovannucci, $2,5,6$ Lorelei A. Mucci, 2,6 Philip W. Kantoff ${ }^{1}$

1 Department of Medicine, Memorial Sloan Kettering Cancer Center, New York, NY

2 Department of Epidemiology, Harvard T.H. Chan School of Public Health, Boston, MA

3 University of North Carolina, Department of Nutrition, Chapel Hill, NC

4 Department of Cancer Epidemiology, Moffitt Cancer Center, Tampa, FL

${ }^{5}$ Department of Nutrition, Harvard T.H. Chan School of Public Health, Boston, MA

${ }^{6}$ Channing Division of Network Medicine, Department of Medicine, Brigham and Women's Hospital, Boston, MA

* These authors contributed equally.

Correspondence: Phil or Konrad

Keywords: CYP27A1, 27-OH-cholesterol, prostate cancer, cholesterol, vitamin D

Prior presentation: Presented in part at the Annual Meeting of the American Association for Cancer Research, Chicago, IL, April 14-18, 2018

Word count: Abstract 251, Manuscript 3040 [with “Harvard"-style citations]

Funding: The Health Professionals Follow-up Study is supported by the National Institutes of Health (U01 CA167552). The Physicians' Health Study was supported by the National Institutes of Health (CA097193, CA34944, CA40360, HL26490, HL34595). The Department of Defense supported K.H.S. (W81XWH-18-1-0330) and P.W.K. (W81XWH-14-1-0515). This research was funded in part by the Dana-Farber/Harvard Cancer Center Specialized Programs of Research Excellence program in Prostate Cancer 5P50 CA090381 and the NIH/NCl Cancer Center Support Grants P30 CA008748 and P30 CA06516. K.H.S. and L.A.M. are Prostate Cancer Foundation Young Investigators.

Acknowledgments: We would like to thank the participants and staff of the Health Professionals Follow-up Study and the Physicians' Health Study for their valuable contributions. In particular, we would like to recognize the contributions of Liza Gazeeva, Siobhan Saint-Surin, Robert Sheahan, and Betsy Frost-Hawes. We would like to thank the following state cancer registries for their help: $A L, A Z$, AR, CA, CO, CT, DE, FL, GA, ID, IL, IN, IA, KY, LA, ME, MD, MA, MI, NE, NH, NJ, NY, NC, ND, OH, OK, OR, PA, RI, SC, TN, TX, VA, WA, WY. The authors assume full responsibility for analyses and interpretation of these data.

Conflicts of interest: The authors declare no potential conflicts of interest. 


\begin{abstract}
Background: Higher intratumoral cholesterol synthesis is associated with a worse prognosis in prostate cancer. The vitamin D-regulated enzyme sterol-27-hydroxylase (CYP27A1) converts cholesterol to 27-hydroxycholesterol, a potential endogenous selective estrogen receptor modulator (SERM). We hypothesized that low CYP27A1 expression is associated low vitamin D signaling, high cholesterol synthesis, and higher risk of lethal prostate cancer.

Methods: We studied 404 patients in the prospective prostate cancer cohorts within the Health Professionals Follow-up Study (HPFS) and the Physicians' Health Study (PHS). We quantified intratumoral CYP27A1 expression and proxies of cholesterol synthesis and vitamin $D$ signaling using transcriptome profiling and assessed prediagnostic plasma 25-hydroxyvitamin $D(25(\mathrm{OH}) \mathrm{D}, \mathrm{n}=132)$ and intratumoral vitamin $D$ receptor protein expression (VDR, $n=300)$. Lethal disease was defined as prostate cancer mortality or metastases, in contrast to non-lethal disease without metastases after $>8$ years of follow-up since diagnosis.

Results: CYP27A1 expression was lower in tumors with higher Gleason grade and higher expression of the second rate-limiting enzyme of cholesterol synthesis, SQLE. We did not detect consistent associations between CYP27A1 and plasma 25(OH)D, CYP24A1, or intratumoral VDR expression. Lower CYP27A1 was associated with higher risk of lethal cancer in both cohorts (adjusted odds ratio for lowest vs. highest quartile of expression, 3.04; 95\% Cl, 1.46-6.33); this association was attenuated when additionally adjusting for Gleason grade and SQLE (odds ratio, 1.76; 95\% Cl, 0.754.17).
\end{abstract}

Conclusion: Low CYP27A1 expression is associated with higher cholesterol synthesis and a higher risk of lethal disease in prostate cancer. Whether the SERM-properties of 27-hydroxycholesterol underlie these associations requires further study. 


\section{Introduction}

Prostate tissue has long been recognized to contain considerable amounts of cholesterol, particularly when undergoing carcinogenic transformation (Schaffner, 1981). More recently, several studies have suggested that higher serum cholesterol levels are associated with increased risk of advanced stage, higher-grade, or fatal prostate cancer (Batty et al., 2011, Mondul et al., 2011, Platz et al., 2008), while others reported null associations (Jacobs et al., 2012). Higher intratumoral synthesis of cholesterol, as assessed through expression of the second rate-limiting enzyme of cholesterol synthesis, squalene monooxygenase (SQLE), is associated with a higher risk of lethal prostate cancer (Stopsack et al., 2016).

A key metabolite of cholesterol is 27-hydroxycholesterol. In preclinical breast cancer models, added 27-hydroxycholesterol stimulated tumor growth, acting partially as an endogenous selective estrogen receptor modulator (SERM) (Nelson et al., 2013, Umetani et al., 2007, Warner and Gustafsson, 2014). Intriguingly, high expression of the enzyme that synthesizes 27hydroxycholesterol, 27-hydroxylase (CYP27A1), has been reported to be associated with higher tumor grade in breast cancer yet better prognosis (Kimbung et al., 2017, Nelson et al., 2013). In prostate cancer, a recent study reported CYP27A1 expression to be strongly inversely related to Gleason grade (Alfaqih et al., 2017). Moreover, CYP27A1 catalyzes the 25-hydroxylation step of vitamin $\mathrm{D}$, which might have a protective effect in various cancers including prostate cancer (Feldman et al., 2014, Shui and Giovannucci, 2014).

We hypothesized that low CYP27A1 expression, potentially resulting in cholesterol accumulation, occurs in prostate cancers that have higher expression of the cholesterol synthesis pathway. We also hypothesized that low CYP27A1 expression is associated with low vitamin D signaling. To test these hypotheses, we conducted a cross-sectional analysis of two large, well characterized populations of patients with prostate cancer. In a longitudinal design, we tested our hypothesis that low CYP27A1 would be associated with a higher risk of lethal prostate cancer over long-term follow-up.

\section{Methods}

\section{Study populations}

We studied patients who were diagnosed with prostate cancer during follow-up of two prospective cohort studies, the Health Professionals Follow-up Study (HPFS) and the Physicians' Health Study (PHS).

The HPFS enrolled 51,529 male health professionals, aged 40 to 75 years, in 1986 (Giovannucci et al., 2007). Participants have been followed through biannual questionnaires since. The PHS enrolled 29,071 male physicians, aged $\geq 40$ years, in 1982, initially for randomizedcontrolled trials of aspirin (Steering Committee of the Physicians' Health Study Research Group, 1989) and micronutrients (Christen et al., 2000). Blood samples were collected from cancer-free participants in 1982 (PHS) and in 1993-95 (HPFS). Self-reported prostate cancer diagnoses in both cohorts are verified through review of medical records. Tissue from all patients included in this study also underwent centralized pathology review. Patients are followed prospectively for metastases and 
prostate cancer-specific death (lethal cancer; with $98 \%$ completeness in HPFS and $99 \%$ in PHS for adjudication of death causes).

Within the prostate cancer biorepository from HPFS and PHS, we conducted a nested "extreme" case-control study, including of whole-transcriptome profiling of the tumor tissue, which oversampled patients with lethal outcome and those with available blood specimens from before cancer diagnosis (Sinnott et al., 2017).

Participants provided written informed consent. The research was approved by the institutional review boards at Harvard T.H. Chan School of Public Health and Partners Healthcare.

Tumor profiling and plasma levels

For all patients included in this study, we retrieved tumor specimens from cancer diagnosis from the treating hospital. Expert genitourinary pathologists performed centralized histologic re-review, including Gleason grading (Stark (Rider) et al., 2009), and selected high-density tumor areas ( $>80 \%$ tumor cell density). Transcriptome profiling included mRNA expressions of CYP27A1, SQLE, and CYP24A1. Tumor tissue and, if available, adjacent non-cancer prostate tissue, was measured on the Affymetrix GeneChip Human Gene 1.0 ST array (Gene Expression Omnibus: GSE62872), with postprocessing as previously described (Penney et al., 2016).

Plasma 25-hydroxy-vitamin $\mathrm{D}[25(\mathrm{OH}) \mathrm{D}]$ from blood samples before cancer diagnosis was measured as a part of a case-control study nested within HPFS. A radioimmunosorbent assay was used, as previously described (Shui et al., 2012).

Vitamin D receptor (VDR) expression in the cytoplasm and membrane was stained via immunohistochemistry on tissue microarrays. Using a semiautomated quantitative image analysis system, the VDR score was generated as a combination of the relative area positively stained and the intensity of staining, as previously described (Hendrickson et al., 2011). TMPRSS2:ERG status was determined using a genomically-validated ERG immunohistochemistry (Pettersson et al., 2012).

\section{Statistical analysis}

Our analysis plan had two main parts. First, we assessed cross-sectionally how CYP27A1 expression was associated with measures of vitamin $D$ signaling and intratumoral cholesterol synthesis. Second, in a longitudinal analysis, we assessed the association between CYP27A1 at cancer diagnosis and the risk of lethal disease over long-term follow-up. All tests were two-sided.

To assess the associations of 25(OH)D, VDR, SQLE, CYP24A1, ERG, and CYP27A1, we used linear regression. Between CYP27A1 and CYP24A1 expressions, we also calculated Pearson correlation coefficients $r$. Values for 25(OH)D were adjusted for season- and batch, as previously described (Shui et al., 2012); VDR scores were adjusted for differences in mean values between tissue microarrays (Hendrickson et al., 2011). We modeled the predictor in categories and inspected plots to assess for potential non-linear relationships, and we calculated tests for linear trend across quartiles by modeling the category medians (for 25(OH)D and VDR) or category indices (mRNA variables) as ordinal predictors. In a sensitivity analysis, we replaced SQLE as a proxy for cholesterol synthesis activity of the tumor by a summary score of all cholesterol synthesis genes (Stopsack et 
al., 2016). This summary score was the first principal component from principal components analysis of all cholesterol synthesis genes. Higher levels indicated higher expression of the cholesterol synthesis pathway, as 20 of the 21 cholesterol synthesis genes were positively loaded on this principal component.

To assess the association of CYP27A1 expression (modeled in quartiles) and lethal disease, we used logistic regression to estimate odds ratios (OR) and $95 \%$ confidence intervals. Models were additionally adjusted for age (linear), year of diagnosis (categorical: pre-prostate specific antigen [PSA] era, 1982-1988; peri-PSA era, 1989-1993; PSA era, 1994-2005), smoking status (binary: current smoker vs. never/prior smoking), family history of prostate cancer in father or brothers (binary: yes $/ \mathrm{no}$ ), body mass index (categorical: $<25,25-30,>30 \mathrm{~kg} / \mathrm{m}^{2}$ ), and hyperlipidemia (binary: self-report of hyperlipidemia by the health professionals vs. no such report). In separate models, we adjusted for Gleason grade (categorical: 5-6, 3+4, 4+3, 8, 9-10) and SQLE expression (categorical: quartiles). In an exploratory analysis, we assessed the association of CYP27A1 within low and high strata of cholesterol synthesis activity defined by SQLE and the cholesterol signature. Given its strong association with lethal disease specifically in the highest quartile (Stopsack et al., 2017), SQLE in the fourth quartile was considered high; the upper half of the signature was considered high.

\section{Results}

Study populations and tumor characteristics at cancer diagnosis

Baseline characteristics of 254 patients from HPFS and 150 patients from PHS are shown in Table 1. $59 \%$ of patients had pathologically organ-confined cancers (T1/T2 N0 M0), and $59 \%$ were diagnosed in the PSA screening era. $92 \%$ of tumor samples were from radical prostatectomy. Plasma concentrations of 25(OH)D before cancer diagnosis were available for a subset of 132 patients from HPFS. VDR protein expression had been quantified for 300 patients.

Notably, CYP27A1 expression was lower in higher-grade, advanced stage, and ERG-positive cancers (Table 1). Compared to Gleason grade 5-6, tumors with Gleason grade 9-10 had on average 0.73 standard deviations (SD) lower CYP27A1 expression $(95 \% \mathrm{Cl}, 0.38$ to $1.08 \mathrm{SD}$; $\left.p_{\text {trend }}<0.001\right)$. ERG-positive tumors had 0.27 SD lower CYP27A1 expression ( $95 \% \mathrm{Cl}, 0.06$ to 0.47 ) than ERG-negative tumors.

Cross-sectional analysis: Vitamin D signaling, cholesterol synthesis, and CYP27A1 expression We assessed the association of circulating and intratumoral indicators of vitamin $D$ signaling and CYP27A1 mRNA expression. Circulating plasma 25(OH)D was not associated with CYP27A1; the difference in CYP27A1 expression between the lowest quartile of 25(OH)D and the highest quartile was $0.04 \mathrm{SD}\left(95 \% \mathrm{Cl},-0.55\right.$ to 0.46 ; $p_{\text {trend }}=0.42$; Figure $\left.1 . \mathrm{A}\right)$. CYP27A1 expression was also not associated with VDR expression in the tumor; the difference in VDR expression score was $0.20 \mathrm{SD}$ $\left(95 \% \mathrm{Cl},-0.25\right.$ to $0.66 \mathrm{SD}$ ) between the lowest and the highest quartile of CYP27A1 ( $p_{\text {trend }}=0.09$; Figure 1.B). In contrast, we observed a weak positive correlation between CYP27A1 and the expression of the VDR target gene CYP24A1 $(r=0.17 ; 95 \% \mathrm{Cl}, 0.07$ to 0.27 ; Figure 1.C).

To assess the association between intratumoral cholesterol synthesis and CYP27A1, we used the second rate-limiting enzyme of cholesterol synthesis, SQLE, and a score summarizing the 
mRNA expression of all cholesterol synthesis enzymes as proxies. CYP27A1 was lower in tumors with higher SQLE expression; the difference in CYP27A1 between first and fourth quartile of SQLE was $-0.42 \mathrm{SD}\left(95 \% \mathrm{Cl},-0.69\right.$ to $-0.14 ; p_{\text {trend }}=0.002$ across quartiles of SQLE; Figure 1.D). Similar results were observed when we used the summary score instead of SQLE as a proxy for cholesterol synthesis in the tumor, observing a difference in CYP27A1 between first and fourth quartile of the score of $-0.49 \mathrm{SD}\left(95 \% \mathrm{Cl},-0.77\right.$ to $\left.-0.22 ; p_{\text {trend }}=0.001\right)$.

In normal prostate tissue, we also did not observe associations between CYP27A1 expression and plasma 25(OH)D and VDR expression (results not shown). In contrast to tumor tissue, CYP27A1 expression and CYP24A1 expression were not correlated in normal prostate tissue ( $r=0.03 ; 95 \% \mathrm{Cl},-0.11$ to 0.17 ), and there was no statistically significant difference in CYP27A1 expression between the lowest and highest quartiles of SQLE (difference, $-0.15 \mathrm{SD} ; 95 \% \mathrm{CI},-0.54$ to $0.25 ; p_{\text {trend }}=0.44$ )

\section{Longitudinal analysis: CYP27A1 and lethal disease}

Patients were followed a median of 15.3 years for the development of metastases or death from prostate cancer (lethal disease). Lower intratumoral CYP27A1 mRNA expression was associated with a higher risk of lethal disease over long-term follow-up in both cohorts (Table 2). In HPFS, patients with CYP27A1 mRNA expression in the lowest quartile had a 2.64-fold higher risk of lethal disease (95\% Cl, 1.23 to 5.67), compared to patients with CYP27A1 in the highest quartile. In PHS, the OR was $4.65(95 \% \mathrm{Cl}, 0.92$ to 23.5$)$. Combining both cohorts and adjusting for additional baseline characteristics, the OR was $3.04\left(95 \% \mathrm{Cl}, 1.46-6.33 ; p_{\text {trend }}=0.007\right.$ across quartiles of CYP27A1). The association of CYP27A1 and lethal disease remained significant but was attenuated somewhat when additionally adjusting for SQLE (OR for lowest vs. highest quartile of CYP27A1, $2.64 ; 95 \% \mathrm{Cl}, 1.24$ to 5.62 ). Results were similar when adjusting for the summary score of cholesterol synthesis. Additional adjustment for Gleason grade considerably attenuated the association of CYP27A1 and lethal disease (OR, 1.92; $95 \% \mathrm{Cl}, 0.83$ to 4.46 ).

As expected, CYP27A1 expression in tumor-adjacent normal prostate tissue was not associated with lethal disease (OR for lowest vs. highest quartile, $1.50 ; 95 \% \mathrm{Cl}, 0.66$ to 3.44 ; $\left.p_{\text {trend }}=0.24\right)$.

Finally, we assessed if the association of intratumoral CYP27A1 with lethal disease differed within levels of cholesterol synthesis. The association between CYP27A1 and lethal disease appeared to be slightly stronger in patients with low SQLE $\left(p_{\text {interaction }}=0.17\right)$. However, we did not observe a similar pattern when stratifying by the summary score of cholesterol synthesis (Table 2; $p_{\text {interaction }}=0.63$ ).

\section{Discussion}

In this study, we assessed regulators of CYP27A1, which synthesizes 27-hydroxycholesterol from cholesterol, and its associations with long-term prognosis in patients with primary prostate cancer. We found CYP27A1 expression to be low in tumors that had higher expression of cholesterol synthesis enzymes including SQLE. In contrast, we did not detect strong associations between several measures of vitamin D signaling and CYP27A1. Notably, low CYP27A1 expression was 
associated with a higher risk of lethal disease, beyond the elevated risk associated with higher expression of the cholesterol synthesis pathway. These findings advance our understanding of the complex role of cholesterol in prostate cancer and may help inform us about the role of CYP27A1 in cancer more broadly.

We observed a strong inverse relationship between CYP27A1 expression and two different measures of intratumoral cholesterol synthesis, the expression of the second rate-limiting enzyme SQLE as well as all enzymes constituting the cholesterol synthesis pathway. These obsevations suggest that in tumors with activated cholesterol synthesis, hydroxylation of cholesterol to 27hydroxycholesterol is inhibited, perhaps in order to make cholesterol available for other purposes in rapidly dividing cells like cell membrane function. Concordantly, a preclinical study found the addition of 27-hydroxycholesterol to prostate cancer cell lines and xenografts attenuated their growth and decreased the expression of SREBP2, the main transcription factor regulating cholesterol synthesis (Alfaqih et al., 2017). However, discordant experimental results, partially using the same cell line, have been reported as well (Raza et al., 2017).

Despite assessing multiple proxies of vitamin D signaling activity, including plasma $25(\mathrm{OH}) \mathrm{D}$ concentrations, VDR protein expression in tumor tissue, and mRNA expression of the VDR target gene CYP24A1, we did not find consistent evidence that showed CYP27A1 to be strongly related to vitamin $D$ signaling. However, these measures may not have fully captured an effect of exogenous vitamin D on CYP27A1 expression, particularly if vitamin $D$ is 25 -hydroxylated directly within prostate cells without changing plasma $25(\mathrm{OH}) \mathrm{D}$ concentrations. This 25 -hydroxylation step of vitamin $\mathrm{D}$ has indeed been observed in a non-tumor prostate cell line, in which vitamin D also induced CYP27A1 expression (Tokar and Webber, 2005). However, the 25-hydroxylase function of CYP27A1 may not be physiologically relevant in peripheral tissues as the prostate, but rather fulfilled by the microsomal 25-hydroxylase CYP2R1 (Jones et al., 2014). Ultimately, our observations lend no additional support to CYP27A1 expression in prostate cancer tissue being tightly controlled by vitamin $D$ signaling. We also assessed if TMPRSS2:ERG status was associated with CYP27A1 expression. Bidirectional influences between vitamin D signaling, including VDR and CYP24A1, and TMPRSS2:ERG have been reported in prostate cancer cell lines (Kim et al., 2014, Washington and Weigel, 2010), and we previously observated that ERG-positive tumors have higher VDR expression (Hendrickson et al., 2011). In the present study, we only observed a modest association between ERG status and CYP27A1 expression, and CYP24A1 expression did not differ by ERG status (data not shown). In breast cancer, several studies found CYP27A1 expression to be higher in high-grade compared to low-grade cancers (Kimbung et al., 2017, Nelson et al., 2013). In prostate cancer, a relatively strong, inverse relationship with Gleason grade has been reported previously (Alfaqih et al., 2017) and was confirmed by our data. CYP27A1 expression has also been reported to be lower in castration-resistant cancer tissue compared to tissue from castration-sensitive tumors (Tamura et al., 2007). How strongly CYP27A1 expression would be associated with risk of clinically relevant outcomes such as metastases or cancer death was unknown. Our data indicated an appropriately three-fold higher risk of lethal cancer among the $25 \%$ patients with the lowest CYP27A1 expression (first quartile), compared to the $25 \%$ with the highest expression (fourth quartile; Table 2). Given the tight association of CYP27A1 and Gleason grade, it is unsurprising that these estimates were 
attenuated considerably when additionally adjusting for Gleason grade. Our results are supported by a previous study that included a larger set of prostate cancer patients from HPFS and found single nucleotide polymorphisms within CYP27A1 to be associated with the risk of lethal disease (Shui et al., 2012); however, we do not know if and how these single nucleotide polymorphisms influence CYP27A1 mRNA expression.

While CYP27A1 does not appear to be well suited as a prognostic marker, our results are informative for mechanistic studies. In particular, it has been suggested that a large proportion of the association of CYP27A1 expression and prognosis in breast cancer is due to SERM effects of 27hydroxycholesterol (Nelson, 2017). These associations were also most pronounced in premenopausal patients with estrogen receptor-positive tumors (Kimbung et al., 2017). Upregulated cholesterol synthesis and production of 27-hydroxycholesterol in estrogen receptor-positive breast cancer under antiestrogen therapy has been suggested as a mechanism of therapy resistance (Nguyen et al., 2015, Simigdala et al., 2016). In our study, we found a moderately strong association of CYP27A1 with lethal disease, which might suggest that at least in prostate cancer, CYP27A1 and 27-hydroxycholesterol would act to some extent through non-SERM mechanisms. Alternatively, 27hydroxycholesterol might be important to consider as a potential ligand when assessing the role of estrogen receptor beta, which is expressed in at least a subset of prostate tumors (Nanni et al., 2009).

We did not find that the association of CYP27A1 expression and lethal disease differed across levels of cholesterol synthesis enzyme expression (Table 2). This could mean that CYP27A1, or tumor features associated with it, drive cancer progression in addition to cholesterol synthesis. Importantly, absence of a statistical interaction between cholesterol synthesis and CYP27A1 on a multiplicative scale should not be interpreted as evidence against biological interaction between them, but rather as the opposite (Greenland et al., 2008).

It should be noted that we measured mRNA levels of CYP27A1 and not protein expression. In an ecologic analysis of a small number of breast tissue samples before and after atorvastatin exposure, CYP27A1 protein appeared to show changes in the opposite direction than CYP27A1 mRNA (Kimbung et al., 2017). In contrast, in prostate cancer tissue, CYP27A1 protein expression was lost in the tumor epithelium in contrast to normal glands, consistent with observations on the mRNA level (Alfaqih et al., 2017). However, this comparison was also ecological, and we are unaware of a study directly comparing mRNA and protein levels within the same patients. An additional limitation of our study is that only a relatively small subset of patients had prediagnostic plasma samples, which may have contributed to the null results for plasma $25(\mathrm{OH}) \mathrm{D}$.

In summary, we found low intratumoral CYP27A1 mRNA expression to be associated with higher markers of intratumoral cholesterol synthesis, higher Gleason grade, and a higher risk of lethal disease over long-term follow-up. We did not find strong associations of CYP27A1 and circulating $25(\mathrm{OH}) \mathrm{D}$ or with two measures of intratumoral vitamin $\mathrm{D}$ signaling. Future studies should consider estrogen receptor expression when evaluating CYP27A1 in prostate cancer and should ideally attempt to directly measure intratumoral or circulating 27-hydroxycholesterol. Interestingly, serum 27-hydroxycholesterol concentrations were decreased by atorvastatin treatment and by vitamin D supplementation in two small-scale clinical trials among patients with breast cancer (Going 
et al., 2017, Kimbung et al., 2017). It remains to be seen how such interventions might affect intratumoral cholesterol and 27-hydroxycholesterol levels as well as clinical outcomes for patients with prostate cancer. 


\section{References}

Alfaqih MA, Nelson ER, Liu W, Safi R, Jasper JS, Macias E, et al. CYP27A1 Loss Dysregulates Cholesterol Homeostasis in Prostate Cancer. Cancer Res 2017;77(7):1662-73.

Batty GD, Kivimaki M, Clarke R, Davey Smith G, Shipley MJ. Modifiable risk factors for prostate cancer mortality in London: forty years of follow-up in the Whitehall study. Cancer Causes Control 2011;22(2):311-8.

Christen WG, Gaziano JM, Hennekens CH. Design of Physicians' Health Study II--a randomized trial of beta-carotene, vitamins E and $\mathrm{C}$, and multivitamins, in prevention of cancer, cardiovascular disease, and eye disease, and review of results of completed trials. Ann Epidemiol 2000;10(2):125-34.

Feldman D, Krishnan AV, Swami S, Giovannucci E, Feldman BJ. The role of vitamin D in reducing cancer risk and progression. Nat Rev Cancer 2014;14(5):342-57.

Giovannucci E, Liu Y, Platz EA, Stampfer MJ, Willett WC. Risk factors for prostate cancer incidence and progression in the health professionals follow-up study. Int J Cancer 2007;121(7):1571-8.

Going CC, Alexandrova L, Lau K, Yeh CY, Feldman D, Pitteri SJ. Vitamin D supplementation decreases serum 27hydroxycholesterol in a pilot breast cancer trial. Breast Cancer Res Treat 2017.

Greenland S, Lash TL, Rothman KJ. Concepts of Interaction. In: Rothman KJ, Greenland S, Lash TL, editors. Modern Epidemiology. 3rd ed. Philadelphia, PA: Lippincott Williams \& Wilkins; 2008.

Greenland S, Mansournia MA, Altman DG. Sparse data bias: a problem hiding in plain sight. BMJ 2016;352:i1981.

Hendrickson WK, Flavin R, Kasperzyk JL, Fiorentino M, Fang F, Lis R, et al. Vitamin D receptor protein expression in tumor tissue and prostate cancer progression. J Clin Oncol 2011;29(17):2378-85.

Jacobs EJ, Stevens VL, Newton CC, Gapstur SM. Plasma total, LDL, and HDL cholesterol and risk of aggressive prostate cancer in the Cancer Prevention Study II Nutrition Cohort. Cancer Causes Control 2012;23(8):1289-96.

Jones G, Prosser DE, Kaufmann M. Cytochrome P450-mediated metabolism of vitamin D. J Lipid Res 2014;55(1):13-31.

Kim JS, Roberts JM, Bingman WE, 3rd, Shao L, Wang J, Ittmann MM, et al. The prostate cancer TMPRSS2:ERG fusion synergizes with the vitamin D receptor (VDR) to induce CYP24A1 expression-limiting VDR signaling. Endocrinology 2014;155(9):3262-73

Kimbung S, Chang CY, Bendahl PO, Dubois L, Thompson JW, McDonnell DP, et al. Impact of 27-hydroxylase (CYP27A1) and 27hydroxycholesterol in breast cancer. Endocr Relat Cancer 2017;24(7):339-49.

Mondul AM, Weinstein SJ, Virtamo J, Albanes D. Serum total and HDL cholesterol and risk of prostate cancer. Cancer Causes Control 2011;22(11):1545-52.

Nanni S, Benvenuti V, Grasselli A, Priolo C, Aiello A, Mattiussi S, et al. Endothelial NOS, estrogen receptor beta, and HIFs cooperate in the activation of a prognostic transcriptional pattern in aggressive human prostate cancer. J Clin Invest 2009;119(5):1093-108.

Nelson ER. The significance of cholesterol and its metabolite, 27-hydroxycholesterol in breast cancer. Mol Cell Endocrinol 2017

Nelson ER, Wardell SE, Jasper JS, Park S, Suchindran S, Howe MK, et al. 27-Hydroxycholesterol links hypercholesterolemia and breast cancer pathophysiology. Science 2013;342(6162):1094-8.

Nguyen VT, Barozzi I, Faronato M, Lombardo Y, Steel JH, Patel N, et al. Differential epigenetic reprogramming in response to specific endocrine therapies promotes cholesterol biosynthesis and cellular invasion. Nat Commun 2015;6:10044.

Penney KL, Pettersson A, Shui IM, Graff RE, Kraft P, Lis RT, et al. Association of Prostate Cancer Risk Variants with TMPRSS2:ERG Status: Evidence for Distinct Molecular Subtypes. Cancer Epidemiol Biomarkers Prev 2016;25(5):745-9.

Pettersson A, Graff RE, Bauer SR, Pitt MJ, Lis RT, Stack EC, et al. The TMPRSS2:ERG rearrangement, ERG expression, and prostate cancer outcomes: a cohort study and meta-analysis. Cancer Epidemiol Biomarkers Prev 2012;21(9):1497-509. 
Platz EA, Clinton SK, Giovannucci E. Association between plasma cholesterol and prostate cancer in the PSA era. Int J Cancer 2008;123(7):1693-8.

Raza S, Meyer M, Goodyear C, Hammer KDP, Guo B, Ghribi O. The cholesterol metabolite 27-hydroxycholesterol stimulates cell proliferation via ERbeta in prostate cancer cells. Cancer Cell Int 2017;17:52.

Schaffner CP. Prostatic cholesterol metabolism: regulation and alteration. Prog Clin Biol Res 1981;75A:279-324.

Shui I, Giovannucci E. Vitamin D status and cancer incidence and mortality. Adv Exp Med Biol 2014;810:33-51.

Shui IM, Mucci LA, Kraft P, Tamimi RM, Lindstrom S, Penney KL, et al. Vitamin D-related genetic variation, plasma vitamin D, and risk of lethal prostate cancer: a prospective nested case-control study. J Natl Cancer Inst 2012;104(9):690-9.

Simigdala N, Gao Q, Pancholi S, Roberg-Larsen H, Zvelebil M, Ribas R, et al. Cholesterol biosynthesis pathway as a novel mechanism of resistance to estrogen deprivation in estrogen receptor-positive breast cancer. Breast Cancer Res 2016;18(1):58.

Sinnott JA, Peisch SF, Tyekucheva S, Gerke T, Lis R, Rider JR, et al. Prognostic Utility of a New mRNA Expression Signature of Gleason Score. Clin Cancer Res 2017;23(1):81-7.

Stark (Rider) JR, Perner S, Stampfer MJ, Sinnott JA, Finn S, Eisenstein AS, et al. Gleason score and lethal prostate cancer: does 3 $+4=4+3$ ? J Clin Oncol 2009;27(21):3459-64.

Steering Committee of the Physicians' Health Study Research Group. Final report on the aspirin component of the ongoing Physicians' Health Study. N Engl J Med 1989;321(3):129-35.

Stopsack KH, Gerke TA, Andren O, Andersson SO, Giovannucci EL, Mucci LA, et al. Cholesterol uptake and regulation in highgrade and lethal prostate cancers. Carcinogenesis 2017;38(8):806-11.

Stopsack KH, Gerke TA, Sinnott JA, Penney KL, Tyekucheva S, Sesso HD, et al. Cholesterol Metabolism and Prostate Cancer Lethality. Cancer Res 2016;76(16):4785-90.

Tamura K, Furihata M, Tsunoda T, Ashida S, Takata R, Obara W, et al. Molecular features of hormone-refractory prostate cancer cells by genome-wide gene expression profiles. Cancer Res 2007;67(11):5117-25.

Tokar EJ, Webber MM. Chemoprevention of prostate cancer by cholecalciferol (vitamin D3): 25-hydroxylase (CYP27A1) in human prostate epithelial cells. Clin Exp Metastasis 2005;22(3):265-73.

Umetani M, Domoto H, Gormley AK, Yuhanna IS, Cummins CL, Javitt NB, et al. 27-Hydroxycholesterol is an endogenous SERM that inhibits the cardiovascular effects of estrogen. Nat Med 2007;13(10):1185-92.

Warner M, Gustafsson JA. On estrogen, cholesterol metabolism, and breast cancer. N Engl J Med 2014;370(6):572-3.

Washington MN, Weigel NL. 1\{alpha\},25-Dihydroxyvitamin D3 inhibits growth of VCaP prostate cancer cells despite inducing the growth-promoting TMPRSS2:ERG gene fusion. Endocrinology 2010;151(4):1409-17. 
Table 1. Characteristics of prostate cancer patients from the Health Professionals Follow-up Study (HPFS) and the Physicians' Health Study (PHS), by CYP27A1 mRNA expression in tumor tissue. Within each quartile, absolute counts (out of 101 patients) closely approximate percentages.

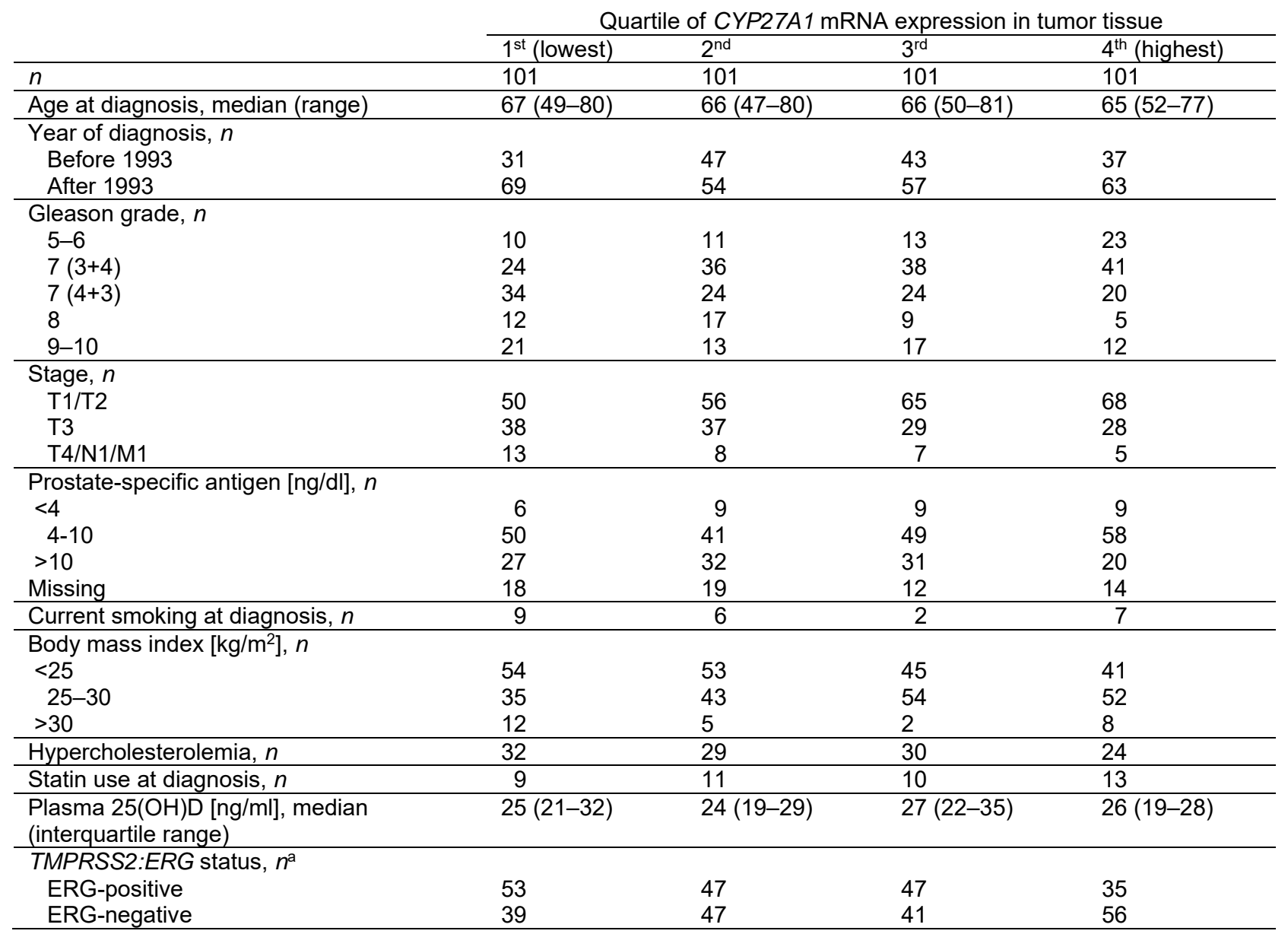

a Based on immunohistochemistry for ERG protein. Missing for 39 patients in total. 
Table 2. CYP27A1 mRNA expression in tumor tissue and lethal prostate cancer.

\begin{tabular}{|c|c|c|c|c|c|}
\hline & \multicolumn{4}{|c|}{ Quartile of CYP27A1 mRNA expression in tumor tissue } & \multirow[b]{2}{*}{$p_{\text {trend }}{ }^{2}$} \\
\hline & 1st (lowest) & $2^{\text {nd }}$ & $3^{\text {rd }}$ & $4^{\text {th }}$ (highest) & \\
\hline \multicolumn{6}{|l|}{ HPFS } \\
\hline Cases: lethal, non-lethal, $n$ & 28,34 & 20,45 & 20,44 & 15,48 & \\
\hline OR $(95 \% \mathrm{Cl}),{ }^{\mathrm{b}}$ unadjusted & $2.64(1.23-5.67)$ & $1.42(0.65-3.11)$ & $1.45(0.66-3.19)$ & 1 (reference) & 0.018 \\
\hline \multicolumn{6}{|l|}{ PHS } \\
\hline Cases: Lethal, non-lethal, $n$ & 8,31 & 10,26 & 10,27 & $2,{ }^{c} 36$ & \\
\hline OR $(95 \% \mathrm{Cl}),{ }^{\mathrm{b}}$ unadjusted & $4.65(0.92-23.5)$ & $6.92(1.40-34.3)$ & $6.67(1.35-33.9)$ & 1 (reference) & 0.12 \\
\hline \multicolumn{6}{|l|}{$\begin{array}{l}\text { Combined HPFS and PHS, } \\
\text { OR }(95 \% \mathrm{Cl})^{\mathrm{b}}\end{array}$} \\
\hline Model 1: Unadjusted & $2.73(1.41-5.30)$ & $2.09(1.06-4.10)$ & $2.09(1.06-4.10)$ & 1 (reference) & 0.005 \\
\hline Model 2: Adjusted ${ }^{d}$ & $3.04(1.46-6.33)$ & $2.17(1.04-4.53)$ & $2.40(1.15-5.00)$ & 1 (reference) & 0.007 \\
\hline Model 3: Model $2+S Q L E^{\mathrm{e}}$ & $2.64(1.24-5.62)$ & $2.17(1.03-4.58)$ & $2.30(1.08-4.88)$ & 1 (reference) & 0.022 \\
\hline Model 4: Model $2+$ chol. score & $2.86(1.35-6.05)$ & $2.02(0.95-4.29)$ & $2.40(1.14-5.05)$ & 1 (reference) & 0.015 \\
\hline Model 5: Model 3 + Gleason & $1.76(0.75-4.17)$ & $1.84(0.77-4.41)$ & $2.05(0.87-4.86)$ & 1 (reference) & 0.31 \\
\hline $\begin{array}{l}\text { By cholesterol score }{ }^{f} \\
\text { OR }(95 \% \mathrm{Cl})^{\mathrm{b}}\end{array}$ & & & & & 9 \\
\hline Score $<$ median & $2.95(1.12-7.81)$ & $1.89(0.67-5.41)$ & $1.77(0.65-4.79)$ & 1 (reference) & 0.032 \\
\hline Score $\geq$ median & $2.22(0.89-5.57)$ & $1.79(0.72-4.48)$ & $2.18(0.85-5.60)$ & 1 (reference) & 0.17 \\
\hline
\end{tabular}

a Test for linear trend across quartiles, modelled as ordinal indices

Odds ratios for lethal disease with $95 \%$ confidence intervals

c Because of the few events in the reference category for PHS $(n=2)$, the odds ratios for PHS alone should be interpreted cautiously in light of probable sparse-data bias (Greenland et al., 2016).

d Adjusted for age (linear), year of diagnosis (categorical), smoking status at cancer diagnosis (binary), body mass index (categorical), high serum cholesterol (binary)

e SQLE and summary score of expression levels for all cholesterol synthesis genes were in quartiles

f HPFS and PHS combined; unadjusted estimates within levels of the cholesterol summary score

$g$ Test for multiplicative interaction between CYP27A1 quartile indices (ordinal) and cholesterol summary score (binary) 


\section{Figure Labels}

Figure 1. Regulation of CYP27A1 mRNA expression in tumor issue. A. Plasma 25(OH)D and CYP27A1 mRNA. B. CYP27A1 mRNA and VDR expression in tumor tissue (in dimensionless units). C. CYP27A1 and CYP24A1 mRNA. D. SQLE mRNA and CYP27A1 mRNA. All units of mRNA expression are standard deviations.

A

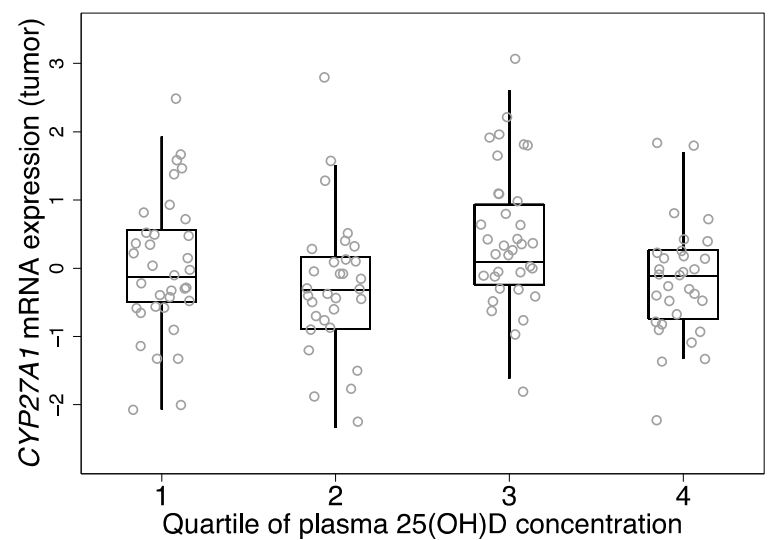

C

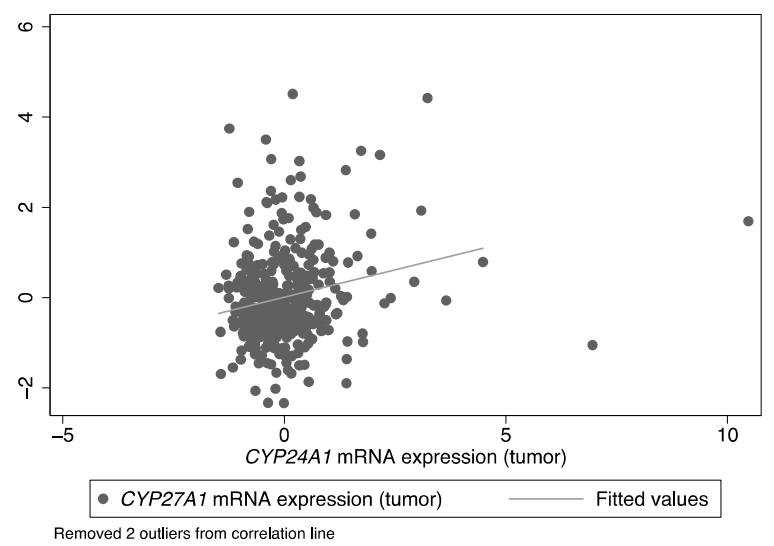

B

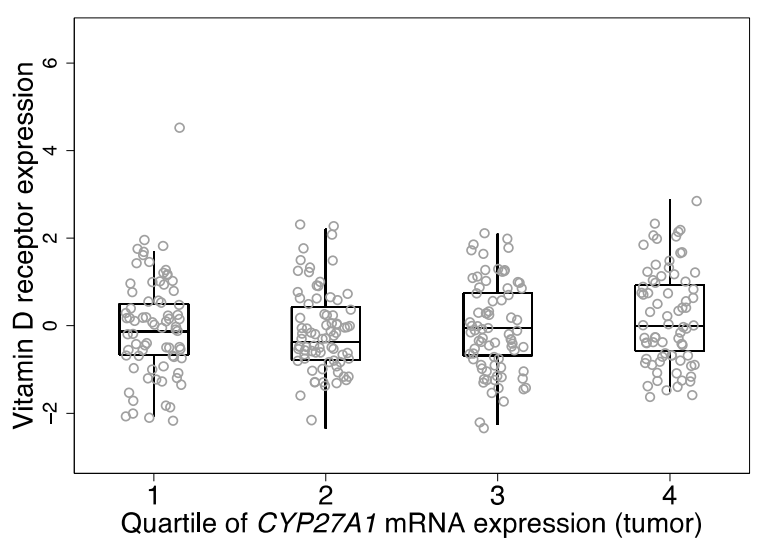

D

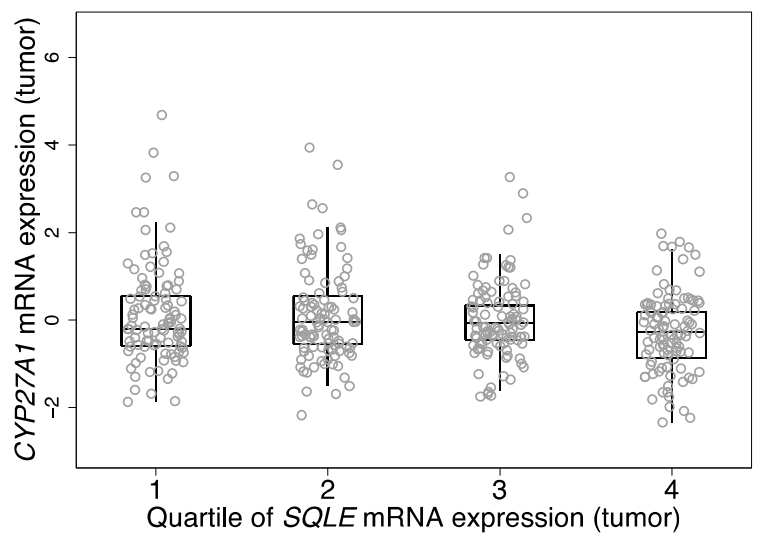

Figure 2. Causal diagram highlighting the complex interplay between cholesterol synthesis, vitamin D signaling, and CYP27A1 in prostate cancer. Note that the graph should be considered incomplete from causal inference standpoint (i.e., it does not list all confounders necessary to estimate causal effects).

[appropriate for readership?] 